\title{
PROBLEMS OF THE Pc1 MAGNETOSPHERIC WAVE THEORY. A REVIEW
}

\author{
A.V. Guglielmi \\ Schmidt Institute of Physics of the Earth RAS, \\ Moscow, Russia, guglielmi@mail.ru
}

\author{
A.S. Potapov \\ Institute of Solar-Terrestrial Physics SB RAS, \\ Irkutsk,Russia,potapov@iszf.irk.ru
}

\begin{abstract}
The Pc1 ultralow-frequency electromagnetic waves (frequency range $0.2-5 \mathrm{~Hz}$ ), also known as pearl necklace, are a unique phenomenon in near-Earth space physics. Many properties of pearls remain a mystery, despite the research of prominent cosmophysicists for more than half a century. In the proposed review, we briefly outline the main points of the so-called standard model, which is widely used to interpret Pc1. Next, we focus on the criticism of the standard model and on the identification of open problems in the Pc1 theory. The general conclusion is that it is necessary to develop new ideas outside the framework of the standard model in
\end{abstract}

\section{INTRODUCTION}

Our review focuses on the so-called pearl necklace one of the most mysterious wave phenomena in near-Earth space. Pearl waves were discovered by E. Sucksdorff and L. Harang at Sodankylä and Tromso observatories in the mid-1930s (see [Kangas et al., 1998; Raita, Kultima, 2007]). In the systematics of ultralow-frequency (ULF) electromagnetic waves of natural origin, pearl waves are classified as Pc1 (0.2-5 Hz frequency range) [Troitskaya, 1964]. The general concept of the origin of Pc1 is that these oscillations are generated in the outer radiation belt and propagate to Earth's surface along geomagnetic field lines [Troitskaya, Guglielmi, 1967].

The Figure provides insight into the dynamic oscillation spectrum. The event shown in the Figure occurred before local midnight. The signal was recorded at low geomagnetic activity $\left(K_{\mathrm{p}}=1_{+}\right)$from the output of the induction magnetometer LEMI-30 installed in the highlatitude complex magnetic-ionospheric station Norilsk (geomagnetic coordinates $\Phi=65.3^{\circ}, \Lambda=162.9^{\circ}$ ). The series of oscillations consists of a quasi-periodic sequence of structural elements. For brevity, they are sometimes referred to as risings. In this case, the average period of repetition of risings is $\sim 160 \mathrm{sec}$. The duration of the series is about an hour; its carrier frequency almost does not change over time, rising only slightly from $0.50 \mathrm{~Hz}$ at the beginning of the series to $0.56 \mathrm{~Hz}$ by its end.

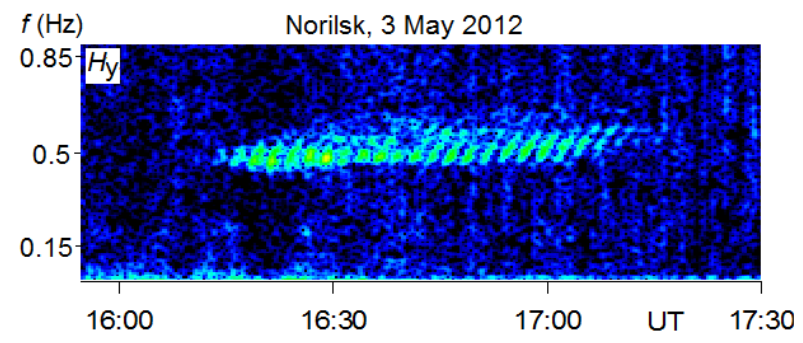

Dynamic spectrum of Pc1 ULF waves order to understand the processes of excitation and propagation of Pc1 waves in Earth's magnetosphere.

Keywords: ultralow-frequency electromagnetic waves, radiation belt, ion-cyclotron resonator, self-excitation mode.

The amazing properties of amplitude-frequency modulation of oscillations, which give a unique originality to the pearl necklace, have attracted the attention of space physicists since the mid-1960s [Yanagihara, 1963; Cornwall, 1965; Tepley, 1965; Troitskaya, Guglielmi, 1967]. Calculations and purposeful observations facilitated the development of the standard model of Pc1 wave excitation and propagation, which became widely known (see, e.g., [Guglielmi, Troitskaya, 1973; Guglielmi, 1979; Nishida, 1980; Guglielmi, Pokhotelov, 1996]). In the first two sections of this paper, we briefly describe the fundamental principles of the standard model.

Over the years, however, internal contradictions of the standard Pc1 model and its incompatibility with the experiment became apparent. Finally, by the beginning of this millennium, a question was thrown into sharp relief about the need for a radical revision of the standard model [Guglielmi et al., 2000]. In the third section, we describe this dramatic situation. It has attracted the attention of many researchers. The problems associated with explaining the Pc1 origin are discussed, for example, in a special issue of a well-known international journal [Kangas et al., 2007], in which different hypotheses about the origin of pearls are compared and critically analyzed [Demekhov, 2007], ground and satellite Pc1 observations are compared [Mursula, 2007], the interaction of Pc1 waves with oscillations of other types [Zolotukhina, Cao, 2007; Kurazhkovskaya et al., 2007] and precipitating charged particles [Yahnin, Yahnina, 2007] is examined. The interest in the mysteries of pearls has not waned with time. In recent years, particular attention has been paid to satellite observations [Paulson et al., 2014; 2017] and their theoretical interpretation [Klimushkin et al., 2010; Mikhailova, 2014; Nekrasov, Feygin, 2018].

In the fourth section, we discuss the problem of pearls in terms of the phenomenological theory of critical phenomena. Our general conclusion is that it is necessary to develop new concepts beyond the standard 
model in order to understand the mysterious properties of excitation and propagation of Pc1 waves in Earth's magnetosphere.

\section{WAVE PACKET}

The standard Pc1 model is based on the fundamental concept of wave packet propagating in a dispersive nonequilibrium medium. The wave packet $\psi(\mathbf{x}, t)$ occupies a limited volume of space at any given time. By $\psi$ we mean any of nonzero components of the alternating electromagnetic field.

To avoid complexity, we examine the wave packet propagating in the direction of the $x$-axis in a homogeneous infinite, initially conservative medium. For clarity, we can imagine that transverse electromagnetic waves of circular polarization travel along lines of a homogeneous external magnetic field in cold hydrogen plasma. Expand $\psi(\mathbf{x}, t)$ in normal plane waves and choose the initial form of the wave packet as Gaussian with rapidly oscillating filling:

$$
\psi(x, 0)=\varphi_{0} \exp \left[-\left(x / x_{0}\right)^{2}+i k_{0} x\right] .
$$

Here $x$ is the marching variable; $x_{0}$ is the width of the packet. Find the corresponding spectral density

$$
\begin{aligned}
& \psi_{k}(0)=\frac{1}{2 \pi} \int_{-\infty}^{\infty} \psi(x, 0) \exp (-i k x) d x= \\
& =\psi_{0} \exp \left[-\frac{1}{4} x_{0}^{2}\left(k-k_{0}\right)^{2}\right]
\end{aligned}
$$

where $\psi_{0}=\varphi_{0} x_{0} / 2 \sqrt{\pi}$. (We have used the tabulated integral value [Gradshtein, Ryzhik, 1962]). The meaning of the transition to the spectral representation is that when $t>0$ the evolution of waves in the $k$ space is trivial, i.e. each spectral component simply oscillates $\psi_{k}(t)=\psi_{k}(0) \exp [-i \omega(k) t]$, with $\omega(k)$ determined by solving the dispersion equation (for the dispersion equation for plane waves in a cold magnetoactive plasma see the monograph [Ginsburg, 1967]).

Now expand $\omega(k)$ in a series in the vicinity of $k_{0}$, using only the first three terms; make the inverse Fourier transform, and determine the law of wave packet evolution:

$$
\begin{aligned}
& \psi(x, t)=\psi_{0} \exp \left(i k_{0} x-i \omega_{0} t\right) \times \\
& \times \sqrt{\frac{\pi}{\alpha+i \beta t}} \exp \left[-\frac{\left(x-v_{g} t\right)^{2}}{4(\alpha+i \beta t)}\right] .
\end{aligned}
$$

Here $\omega_{0}=\omega\left(k_{0}\right), \quad \alpha=x_{0}^{2} / 4, \beta=\left(d v_{g} / d k\right) / 2$ for $k=k_{0}$. We can see that the wave packet propagates as a whole with the group velocity $v_{g}=d \omega / d k$ and spreads due to dispersion. The fundamental concept of group velocity was introduced independently by Stokes and Rayleigh in the 1870s. At the same time, Umov interpreted $v_{\mathrm{g}}$ as the velocity of wave energy-momentum transfer. If $v_{\mathrm{g}}$ depends on frequency, the medium is said to be dispersive with respect to waves of this type. Note that the term "group velocity" was introduced by Ray- leigh, whereas the term "dispersion" goes back to Newton.

It is easy to verify that the maximum amplitude of the packet traveling with the velocity $v_{\mathrm{g}}$ decreases with time as $\left(\alpha^{2}+\beta^{2} t^{2}\right)^{-1 / 4}$. The packet width increases with time as $\left(\alpha^{2}+\beta^{2} t^{2}\right)^{1 / 2}$. Its total energy remains constant. When $t>0$, the spectral components are redistributed within the packet such that when $x=$ const the instantaneous frequency increases with time if $\beta<0$, and decreases if $\beta>0$.

The wave energy conservation law results from the conservatism of the ideal medium we have chosen as cold magnetoactive plasma. In fact, the waves are absorbed, their energy is converted into heat, but they may also amplify if the medium is nonequilibrium, as is the case in the external radiation belt, where a small addition of energetic ions (mostly protons) creates conditions for the occurrence of ion-cyclotron instability [Cornwall, 1965]. There is, however, a detail that was not immediately included in the structure of the standard Pc1 model [Troitskaya, Guglielmi, 1967; Guglielmi, 1979]. The situation is as follows. In the pioneer work [Cornwall, 1965], the initial perturbation is taken as a monochromatic ion cyclotron wave, which propagates in a homogeneous infinite cold plasma comprising an addition of nonequilibrium energetic ions. But the model of the monochromatic wave filling the infinite space is difficult to adapt, even conceptually, to radiation belt conditions. Moreover, we will show that the radiation belt is not a generator - it is an amplifier of ioncyclotron waves.

Take the initial perturbation in the form of a wave packet, as we have done above, rather than in the form of a monochromatic wave. Replace the frequency $\omega$ in the dispersion equation with the complex value $\omega+i \gamma$ whose imaginary part is commonly referred to as the medium instability increment relative to wave perturbations of this type if $\gamma>0$ or the wave damping decrement if $\gamma<0$. The calculation of $\gamma$ in the framework of realistic medium models is a mathematically complex and often cumbersome procedure. It suffices here to assume that $\gamma>0$ in a certain frequency band $\omega$ (or wave numbers $k$ ), and $\gamma \leq 0$ outside this band.

Consider a low-amplitude wave packet in a nonequilibrium medium. Assume that the $\gamma$ distribution in the instability strip is unimodal, as it happens in many cases (see, e.g., [Guglielmi, Pokhotelov, 1996]). Expand the $\gamma(k)$ and $\omega(k)$ functions in the vicinity of the maximum increment:

$$
\begin{aligned}
& \gamma=\gamma_{m}-a\left(k-k_{m}\right)^{2}, \\
& \omega=\omega_{m}+v_{g}\left(k-k_{m}\right)+b\left(k-k_{m}\right)^{2} .
\end{aligned}
$$

Here $\quad \gamma_{m}=\gamma\left(k_{m}\right), \quad \omega_{m}=\omega\left(k_{m}\right), \quad a=d^{2} \gamma /\left.2 d k^{2}\right|_{k=k_{m}}$, $b=d^{2} \omega /\left.2 d k^{2}\right|_{k=k_{m}}, \gamma_{m}>0, a>0$.

The further calculation, which is quite similar to the above one, gives the following law of wave packet evolution in a nonequilibrium medium: 


$$
\psi(x, t)=\psi_{0}\left[\frac{\pi}{(a+i b) t+x_{0}^{2} / 4}\right]^{1 / 2} \exp \left\{i\left(k_{m} x-\omega_{m} t\right)+\gamma_{m} t-\frac{\left[x-v_{g} t+i x_{0}^{2}\left(k_{m}-k_{0}\right) / 2\right]^{2}}{4(a+i b) t+x_{0}^{2}}-\frac{x_{0}^{2}}{4}\left(k_{m}-k_{0}\right)^{2}\right\} .
$$

The packet amplitude increases exponentially with time due to the fact that the free energy of nonequilibrium plasma is transformed into the wave energy.

Formula (4) allows us to introduce a concept of significant difference between absolute and convective instabilities of nonequilibrium medium, which is important for the Pc1 theory [Lifshitz, Pitaevskii, 1979]. With the absolute instability, the amplitude increases at any space point. For this purpose, as follows from (4), the inequality

$$
\gamma_{m}>\frac{v_{g}^{2} a}{4\left(a^{2}+b^{2}\right)}
$$

should hold. With the inverse inequality, the instability is convective: the amplitude remains finite at any fixed space point. It increases exponentially only in the reference frame moving with the group velocity, for example, at the point $x=v_{\mathrm{g}} t$. Note that for ion-cyclotron waves in the radiation belt the condition of convective instability is almost always fulfilled. This means that in the radiation belt the ion-cyclotron waves may be amplified rather than generated.

\section{STANDARD MODEL}

The self-excitation of ion-cyclotron waves is possible in the expanded system that includes a radiation belt and an open resonator. The resonator is formed by the geomagnetic flux tube with its ends resting on the ionosphere in magnetoconjugated regions of the Northern and Southern hemispheres. The wave packet, amplified in the radiation belt, propagates to Earth, reaches one of the resonator's ends, is partially reflected, arrives at the amplifier input, and then the process is repeated in the opposite hemisphere. If coefficients of reflection from the ionosphere are large enough, the radiation belt begins generating oscillations at the ion-cyclotron resonance frequency

$$
\omega_{m}=\Omega_{\mathrm{p}}\left(c_{\mathrm{A}} / v_{\| \mathrm{p}}\right),
$$

where $\Omega_{\mathrm{p}}$ is the proton gyrofrequency at the equator of the flux tube, $c_{\mathrm{A}}$ is the Alfvén velocity, $v_{\| \mathrm{p}}$ is the longitudinal component of energetic proton velocity. Here, for simplicity, we take the condition $c_{\mathrm{A}} \ll v_{\| \mathrm{p}}$. These are the general characteristics of the standard model of Pc1 ULF wave excitation described in more detail in the reviews and monographs [Troitskaya, Guglielmi, 1967; Guglielmi, Troitskaya, 1973; Guglielmi, Pokhotelov, 1996; Kangas et al., 1998].

In the standard model, the dynamic spectrum of the pearl necklace, presented in the Figure, is interpreted as follows. In fact, we observe the same ion-cyclotron wave packet oscillating along the geomagnetic flux tube, being reflected from the ionosphere in magnetoconjugate regions. The packet periodically returns to the radiation belt, is amplified, and the process repeats. Oscillations are generated provided that the energy inflow from the radiation belt in the wave field exceeds the losses occurring due to wave reflection from the ionosphere. The period of repetition of the structural elements (see Figure) is equal to twice the travel time from one conjugate point to another with the group velocity $v_{g}$. The carrier frequency is determined by resonance condition (6). An increase in the frequency within a single structural element is due to the fact that ion-cyclotron waves suffer dispersion spreading with the dispersion parameter being negative: $\beta=d v_{g} / 2 d k<0$ (see Formula (2)).

We have not specifically mentioned this fact, but it is clear from the context that the linear theory of wave propagation is used for the analysis. In other words, the amplitude of initial perturbation (1) is assumed to be sufficiently small. In an unstable medium, the wave packet is evolving for only a short time according to (4). The packet amplitude increases exponentially, and the linear theory becomes invalid $\sim 1 / \gamma_{m}$ after the start of the process. The detailed analysis of the nonlinear stage of evolution is beyond the scope of this paper. We restrict ourselves to describing one scenario for evolution, which is interesting from a physical point of view and perhaps useful for interpreting observations.

The picture of the evolution of small perturbations in an amplifying medium is qualitatively as follows. At the linear stage, initial perturbations increase with their spectrum narrowing rapidly and concentrating in a small vicinity of the resonance frequency $\omega_{m}=\omega\left(k_{m}\right)$. As the amplitude increases, nonlinear processes come into action. Nonlinearity is called dissipative if $\gamma(k)$ is modified under the action of waves, or conservative if $\omega(k)$ is modified. Focus on the conservative nonlinearity and model it by adding the term $\eta|\psi|^{2}$ to the right-hand side of the second of formulas (3). Assume that the Lighthill criterion $\eta b<0$ is met. Then the modulation instability of wave field arises. This leads to spectral broadening, which in turn leads to wave energy transfer from the amplification range $\left(\left|k-k_{\text {res }}\right|<\Delta k\right)$ to the dissipation range $\left(\left|k-k_{\mathrm{res}}\right|>\Delta k\right)$. Here $\Delta k$ is the amplification bandwidth. Thus, due to the energy transfer along the spectrum, the amplitude $|\psi|$ stabilizes.

We have adopted this scenario from the theory of wind waves on the water surface [Andronov, Fabrikant, 1979]. It might take place in a nonequilibrium plasma too [Guglielmi, 2008]. Let us call your attention to an interesting fact. The modulation instability is believed to give rise to solitons. In an active (amplifying) dispersing nonlinear medium, a soliton appears to have no free parameters. Its amplitude, width, and carrier frequency is completely determined by medium parameters. Recall that a soliton in a passive medium has two free parameters, namely, amplitude and carrier frequency. The width of the soliton is determined by its amplitude. In contrast, in the linear wave packet propagating in a pas- 
sive medium, the amplitude, width, and carrier frequency are independent, i.e. are free parameters (see (2)).

\section{PROBLEMS OF INTERPRETATION}

Beauty, elegance, and depth of the theory of wave packet propagation manifest themselves in the fact that of a small number of postulates we managed to infer a variety of opinions about amazing properties of Pc1 waves. Many of properties of pearls, if not all, seem to be understood and described in terms of the theory, at least qualitatively. But look at the slope of the structural elements in the Figure, it does not change with time. This roughly contradicts the linear theory of wave packet spreading since the theory predicts that the slope of elements must increase monotonically. An original hypothesis about the origin of the anomalous dispersion of pearls was proposed in the framework of the quasilinear theory of plasma instability [Feygin, Yakimenko, 1969, 1970; Feygin, Kurchashov, 1975]. However, the problem cannot be considered solved because without alternative assumptions we cannot experimentally test the hypothesis about the quasilinear mechanism of the formation of pearl dispersion.

Let us refer to the second problem as the discreteness problem. The crux of the problem is that in the linear theory of waves in a dispersive nonequilibrium medium there is no stratification mechanism of dynamic spectrum such as that shown in the Figure. As in the case of the anomalous dispersion, we have to supplement the standard model with the hypothesis on the nonlinear mechanism of the formation of a quasiperiodic sequence of structural elements. In this context, a structural element of pearl series is sometimes spoken of as a soliton, but this is nothing more than a general line of thought when searching for the solution of the discreteness problem. There is no physical content in the analogy between pearls and solitons.

The problems of pearl discreteness and dispersion may be called theoretical in the sense that observations show clear stable properties of oscillations, whereas the theory appears to be insufficiently sophisticated to explain these properties. Moreover, both of these problems seem to be associated with the theory of nonlinear oscillations. In contrast, the third problem, presented below, is experimental and can be fully formulated in the framework of the linear theory of ion-cyclotron wave propagation. We call it the problem of the existence of ion-cyclotron resonators in the magnetosphere (ICR problem). Its crux is that the theory indicates the existence of ICR, but we could not find any experimental evidence of it, even despite our efforts [Guglielmi et al., 2000; Guglielmi et al., 2001; Guglielmi, Potapov, 2012].

The ICR problem arises due to the presence of small addition of heavy ions (e.g., $\mathrm{O}^{+}$) in the magnetosphere. The specific irregularity of the geomagnetic field in the vicinity of the equatorial plane plays a key role in forming ICR. We have, however, agreed not to go to the heart of the complex problem of wave propagation in inhomogeneous media. We therefore restrict ourselves to a brief comment and the above references (see also the review [Guglielmi, 2007]).
The main difference between ICR and the resonator of the standard model of pearl self-excitation is that wave reflection points are not in the ionosphere but high above Earth's surface, in a small vicinity of the geomagnetic field line vertex representing the axis of the resonator. In this regard, there is an additional problem of interpretation since it is unclear how the energy of waves accumulated in ICR is transferred to Earth.

It is interesting that the oscillation period of the wave packet between ICR ends is approximately equal to the period of repetition of structural elements of pearl series. Moreover, the linear wave packet in ICR does not spread, although it propagates in the dispersive medium. This partially solves the first of the said problems of interpretation. It is still not quite clear why we usually observe signals of a rising tone rather than, say, of a falling one. The concept of ICR, on the existence of which the theory provides strong evidence, has allowed us to understand one of the fundamental properties of pearls - a clear anticorrelation of occurrence of oscillations of this type with the 11-year solar cycle, namely, we managed to relate this regularity with the 11-year solar cycle variation of the density of heavy $\mathrm{O}^{+}$ions in Earth's magnetosphere [Guglielmi et al., 2001; Guglielmi, 2007]. At the same time, the ICR theory as linear gives no evidence for the stratification of the dynamic spectrum, clearly visible in the Figure.

\section{DISCUSSION}

We have seen how difficult it may be to interpret electromagnetic ULF waves of natural origin. Dozens of space physicists armed with the highly advanced theory of wave propagation in dispersive nonequilibrium media have not unraveled the puzzle of pearls even after the extensive research carried out for more than half a century. We deem the discreteness problem to be the most difficult. To find a way to solve the problem, we turn to the theory of critical phenomena and start with the Landau phenomenological theory, adapting it to our case.

Introduce a parameter of order of $\varepsilon=|\psi|^{2}$ proportional to the wave energy density at the center of ICR, averaged, if necessary, over a certain time interval. The evolution equation has the form

$$
\frac{d \varepsilon}{d t}=2 \Gamma \varepsilon .
$$

The nonlinear increment $\Gamma(\Lambda, \varepsilon)$ is

$$
\Gamma(\Lambda, \varepsilon)=\gamma(\Lambda)-\alpha \varepsilon,
$$

where $\alpha$ is the Landau constant, $\Lambda$ is the controlling parameter [Landau, Lifshitz, 1988] (the Landau parameter should not be confused with the parameter $\alpha$ in Formula (2)). Near the self-excitation threshold, the linear increment $\gamma(\Lambda)$ is proportional to the controlling parameter:

$$
\gamma(\Lambda)=\beta\left(\Lambda-\Lambda_{\mathrm{c}}\right)
$$

Here $\Lambda_{c}$ is the critical value of $\Lambda ; \beta$ is the proportionality factor. The theory of ion-cyclotron instability of the radiation belt [Cornwall, 1965; Kangas et al., 1998] suggests selecting the controlling parameter $\Lambda=N\left(T_{\perp} / T_{\|}-1\right)$. Here $N$ is the energetic proton density; 
$T_{\|}, T_{\perp}$ are the longitudinal and transverse proton temperatures. Thus, the theory has three phenomenological parameters $-\alpha, \beta, \Lambda_{c}$.

In equilibrium $d \varepsilon / d t=0$. Equilibrium may be disordered when $\varepsilon=0$ or ordered when $\varepsilon \neq 0$ at $\Gamma(\varepsilon, \Lambda)=0$. The sign of $\alpha$ is important in expression (8) for the nonlinear increment. If $\alpha>0$, the dynamic system is said to have soft self-oscillation; and if $\alpha<0$, hard selfoscillation. The quasilinear theory of interaction between waves and particles in the radiation belt predicts that $\alpha>0$. Apparently, in the soft mode it is difficult to expect the pearl discreteness to form. In fact, the system with the soft self-oscillation at $\Lambda<\Lambda_{\mathrm{c}}$ features a disordered state; and at $\Lambda>\Lambda_{c}$, an ordered state. In the latter case, oscillations are excited by infinitely small perturbations and there are no intervals between risings in the pearl necklace.

In this respect, the hard self-oscillation mode would be more interesting [Guglielmi, Troitskaya 1973]. Unlike the dynamic system with soft self-oscillation, the system with hard self-oscillation is metastable in a subcritical state. In other words, it is stable with respect to infinitely small perturbations, but can be transformed into the self-oscillating mode under the influence of a trigger, i.e. into perturbations with small but finite amplitude. However, specific mechanisms for the hard self-oscillation mode in the radiation belt have not been found yet.

We think that the development of the theory based on the concept of flow-type resonator is also interesting and promising. It was proposed by Guglielmi [1971] The case in point was the resonator of standard model of pearls, but the concept of flowage can also be applied to ICR. The situation is as follows. Side walls of the open resonator are formed by a caustic system, i.e. they are transparent for radiation belt ions drifting from east to west in the inhomogeneous geomagnetic field. Thus, ICR is blown off by a stream of hot particles having a certain free energy. Outside the resonator, the free energy is not transformed into the wave energy since, as we have noted earlier, the radiation belt particle distribution instability is convective. However, inside the resonator such transformation is possible and under certain conditions waves are self-excited. The quasilinear interaction between waves and particles in ICR leads to a reduction in free energy, but the inflow of new particles compensates for the losses. The current nonlinear increment $\Gamma$ in the flow-type resonator is appeared to depend on the prehistory of wave field evolution. This circumstance is formally expressed as follows:

$$
\Gamma(\Lambda, \varepsilon, t)=\gamma(\Lambda)-\eta \int_{-\infty}^{t} \varepsilon\left(t^{\prime}\right) \Phi\left(t^{\prime}-t\right) d t^{\prime} .
$$

Guglielmi [1971] shows that the wave field is partitioned into a periodical sequence of discrete signals if the form factor $\Phi=0$ when $t^{\prime}<t-\tau$ and $\Phi=1$ at $t-\tau \leq t^{\prime} \leq t$. Here $\tau$ is the time of transport of radiation belt energetic particles across ICR. According to an approximate estimate, $\tau$ coincides with the pearl-repetition period.

The concept of the flow-type resonator was taken up by other researchers and was developed in a number of papers [Bespalov, Trakhtengertz, 1986; Tagirov et al.,
1986; Trakhtengertz et al., 1986; Trakhtengertz, Demekhov, 2002] at the phenomenological level. An effective microscopic theory has not been developed, so the question about the mechanism of the formation of pearl discreteness remains open. What is clear is that the discreteness, which was noted by Sucksdorff in his pioneer paper [Sucksdorff, 1936], is incompatible with the standard theory of origin of pearls.

To conclude this section, we present a speculative argument that may give some insight into the origin of risings in a series of pearls (see Figure). Suppose that for whatever reason $\Gamma=0, \varepsilon=0$ at $t=0$. This can occur as a result of wave field evolution in accordance with Equations (7) and (10). At $t=0$, no self-oscillation occurs. However, at $t>0$ new energetic radiation belt ions drifting from east to west and having an excess free energy begin to arrive at the resonator (this is the manifestation of ICR flowage). As the resonator is filled, the self-oscillation occurs again. It is quite clear that more energetic particles fill ICR earlier than the less energetic ones. But this means that the oscillations at a rather low frequency are excited earlier than those at higher frequency in accordance with resonance condition (6). In other words, the frequency increase in the structural element is not associated with wave packet spreading, but with the spread of radiation belt particles in velocity. It is easy to verify that $\omega \propto \sqrt{t}$, i.e. the function $\omega(t)$ is convex within a rising, which is usually observed in practice.

\section{CONCLUSION}

We have analyzed open problems of physics of magnetospheric Pc1 waves and have verified that it is necessary to develop new concepts beyond the standard model in order to understand the amazing properties of the dynamic oscillation spectrum.

Generally speaking, the expansion of the range of the problems indicates successful development of a particular field of research. However, the said Pc1 problems have remaining open for so long that they begin to cause concern. We are sure that due to collective efforts the mechanism of the formation of stable wave structures observed on Earth and in space as a pearl necklace will ultimately be understood. We pin our hopes primarily on the concept of the flow ion-cyclotron resonator, located in the equatorial region of the outer radiation belt.

We are grateful to B.V. Dovbnya, O.D. Zotov, B.I. Klain, and F.Z. Feygin for their interest in this work. This work was performed with budgetary funding of RFBR (project No. 19-05-00574), project of the Ministry of Education and Science of the Russian Federation KP19-270, as well as programs of state task of IPE RAS (No. 0144-2014-00116) and ISTP SB RAS (II.16). Results were partially obtained using the equipment of Center for Common Use "Angara" http://ckprf.ru/ckp/3056/.

\section{REFERENCES}

Andronov A.A., Fabrikant A.L. Landau damping, wind waves and whistle. Nelinejnye volny [Non-Linear Waves]. Moscow, Nauka Publ., 1979, pp. 68-104. (In Russian). 
Bespalov P.A., Trakhtengertz V.Yu. Alvenovskiye mazery [Alfven Masers]. Gorky, IPPh AS USSR, 1986, 190 p. (In Russian).

Cornwall J.M. Cyclotron instabilities and electromagnetic emission in the ultralow frequency and very low frequency ranges. J. Geophys. Res. 1965, vol. 70, pp. 61-69.

Demekhov A.G. Recent progress in understanding Pc1 pearl formation. J. Atmos. Solar-Terr. Phys. 2007, vol. 69, pp. 1609-1622. DOI: 10.1016/j.jastp.2007.01.014.

Feygin F.Z., Kurchashov Yu.P. A quasilinear dynamics of Pc1 geomagnetic pulsations (Pearls). J. of Geomagnetism and Geoelectricity. 1975, vol. 26, pp. 539-548.

Feygin F.Z., Yakimenko V.L. The mechanism of generation and development of "pearls" under the cyclotron instability of the outer proton zone. Geomagnetizm $i$ aeronomiya [Geomagnetism and Aeronomy]. 1969, vol. 9, no. 4, pp. 700705. (In Russian).

Feygin F.Z., Yakimenko V.L. On the fine structure of micropulsations of the Pc1 type. Geomagnetizm $i$ aeronomiya [Geomagnetism and Aeronomy]. 1970, vol. 10, no. 3, pp. 558-560. (In Russian).

Ginzburg V.L. Rasprostranenie elektromagnitnykh voln v plazme [Propagation of electromagnetic waves in plasma]. Moscow, Nauka Publ., 1967, 684 p. (In Russian).

Gradshtein I.S., Ryzhik I.M. Table of integrals, series, and products. New York, Academic Press, 1965, 1086 p.

Guglielmi A.V. Cyclotron instability of the outer radiation belt of the Earth under conditions of self-modulation of growing waves. JETP Lett. 1971, vol. 13, no. 2, pp. 85-88. (In Russian).

Guglielmi A.V. MGD-volny v okolozemnoi plazme [MHD Waves in the Near-Earth Plasma]. Moscow, Nauka Publ., 1979, 139 p. (In Russian).

Guglielmi A.V. Ultralow-frequency electromagnetic waves in the Earth's crust and magnetosphere. PhysicsUspekhi. 2007, vol. 50, no. 12, pp. 1197-1216.

Guglielmi A.V. Nonlinearity of geoelectromagnetic waves. Geofizicheskie issledovaniya [Geophys. Res.]. 2008 vol. 9, no. 3, pp. 16-24. (In Russian).

Guglielmi A.V., Pokhotelov O.A. Geoelectromagnetic Waves. IOP Publ. Ltd. Bristol and Philadelphia, 1996, 402 p.

Guglielmi A.V., Potapov A.S. The effect of heavy ions on the spectrum of oscillations of the magnetosphere. Cosmic Res. 2012, vol. 50, no. 4, pp. 263-271. DOI: 10.1134/S0010952512040016.

Guglielmi A.V., Troitskaya V.A. Geomagnitnye pulsatsii i diagnostika magnitosfery [Geomagnetic pulsations and diagnostics of the magnetosphere]. Moscow, Nauka Publ., 1973, 208 p. (In Russian).

Guglielmi A., Potapov A., Russell C. The ion cyclotron resonator. JETP Lett. 2000, vol. 72, pp. 432-435.

Guglielmi A., Kangas J., Potapov A. Quasi-periodic modulation of the Pc1 geomagnetic pulsations: An unsettled problem. $J$. Geophys. Res. 2001, vol. 106, no. A11, pp. 25847-25856.

Kangas J., Guglielmi A., Pokhotelov O. Morphology and physics of short-period magnetic pulsations (A Review). Space Sci. Rev. 1998, vol. 83, pp. 435-512.

Kangas J., Fraser B., Potapov A. Pc1 pearl waves: Discovery, morphology and physics. Preface. J. Atmos. Solar-Terr. Phys. 2007, vol. 69, pp. 1599. DOI: 10.1016/j.jastp.2007.06.004

Klimushkin D.Yu., Mager P.N., Marilovtseva O.S. Parallel structure of Pc1 ULF oscillations in multi-ion magnetospheric plasma at finite ion gyrofrequency. J. Atmos. Solar-Terr. Phys. 2010, vol. 72, pp. 1327-1332. DOI: 10.1016/j.jastp.2010.09.019.

Kurazhkovskaya N.A., Klain B.I., Dovbnya B.V. Patterns of simultaneous observations of high-latitude magnetic impulses (MIEs) and impulsive bursts in the Pc1-2 band. $J$. Atmos. Solar-Terr. Phys. 2007, vol. 69, pp. 1680-1689. DOI 10.1016/j.jastp.2006.12.003.

Landau L.D., Lifshitz E.M. Gidrodinamika [Hydrodynamics]. Moscow: Nauka Publ., 1988, 733 p. (In Russian).
Lifshitz E.M., Pitaevskii L.P. Physical Kinetics. Course of Theoretical Physics, vol. 10 (1 ed.). Oxford: Pergamon, 1981, $453 \mathrm{p}$.

Mikhailova O.S. The spatial structure of ULF-waves in the equatorial resonator localized at the plasmapause with the admixture of the heavy ions. J. Atmos. Solar-Terr. Phys. 2014, vol. 108, pp. 10-16. DOI: 10.1016/j.jastp.2013.12.007.

Mursula K. Satellite observations of Pc1 pearl waves: The changing paradigm. J. Atmos. Solar-Terr. Phys. 2007, vol. 69, pp. 1623-1634. DOI: 10.1016/j.jastp.2007.02.013.

Nekrasov A.K., Feygin F.Z. Ponderomotive force of ion cyclotron waves in the Pc1 frequency range with magnetosonic dispersion. Izvestiya. Physics of the Solid Earth. 2018, vol. 54, no. 5, pp. 741-748.

Nishida A. Geomagnetic Diagnosis of the Magnetosphere. New York, Springer-Verlag, 1978, 2013, 256 p.

Paulson K.W., Smith C.W., Lessard M.R., Engebretson M.J., Torbert R.B., Kletzing C.A. In situ observations of Pc1 pearl pulsations by the Van Allen Probes. Geophys. Res. Lett. 2014, vol. 41, pp. 1823-1829. DOI: 10.1002/2013GL059187.

Paulson K.W., Smith C.W., Lessard M.R., Torbert R.B., Kletzing C.A., Wygant J.R. In situ statistical observations of Pc1 pearl pulsations and unstructured EMIC waves by the Van Allen Probes. J. Geophys. Res.: Space Phys. 2017, vol. 122, pp. 105-119. DOI: 10.1002/2016JA023160.

Raita T., Kultima J. Discovery of the pearl waves by Eyvind Sucksdorff. J. Atmos. Solar-Terr. Phys. 2007, vol. 69, pp. 1600-1603. DOI: 10.1016/j.jastp.2007.01.013.

Sucksdorff E. Occurences of rapid micropulsations at Sodankylä during 1932 to 1935. Terrestrial Magnetism and Atmospheric Electricity. 1936, vol. 41, pp. 337-344.

Tagirov V.R., Trakhtengertz V.Yu., Chernous S.A. On the nature of aurora pulsing spots. Geomagnetizm i aeronomiia [Geomagnetism and Aeronomy]. 1986, vol. 26, no. 4, pp. 600-604. (In Russian).

Tepley L. Regular oscillations near $1 \mathrm{c} / \mathrm{s}$ observed simultaneously at middle and low latitudes. Radio Sci. 1965, vol. 69D, pp. 1089-1105.

Trakhtengertz V.Yu., Demekhov A.G. Cosmic cyclotron masers. Priroda [Nature]. 2002, no. 4, pp. 25-31. (In Russian).

Trakhtengertz V.Yu., Tagirov V.R., Chernous S.A. A flowthrough cyclotron maser and pulsed VLF emission. Geomagnetizm i aeronomiia [Geomagnetism and Aeronomy]. 1986, vol. 26, no. 1, pp. 99-106. (In Russian).

Troitskaya V.A. Classification of rapid pulsations of the magnetic field and earth currents. Geomagnetism and Aeronomy. 1964, vol. 4, p. 490-491.

Troitskaya V.A., Guglielmi A.V. Geomagnetic micropulsations and diagnostics of the magnetosphere. Space Sci. Rev. 1967, vol. 7, no. 5/6, pp. 689-769.

Yahnin A.G., Yahnina T.A. Energetic proton precipitation related to ion-cyclotron waves. J. Atmos. Solar-Terr. Phys. 2007, vol. 69, pp. 1690-1706. DOI: 10.1016/j.jastp.2007.02.010.

Yanagihara K. Geomagnetic micropulsations with periods from 0.03 to 10 seconds in auroral zones with special reference to conjugate-point studies. J. Geophys. Res. 1963, vol. 68 , no. 11 , pp. 3383-3397.

Zolotukhina N., Cao J. Transformation of structured Pc1 into IPDP-like emission under enhanced magnetospheric convection: A case study. J. Atmos. Solar-Terr. Phys. 2007, vol. 69, pp. 1668-1679. DOI: 10.1016/j.jastp.2007.01.016.

\section{How to cite this article}

Guglielmi A.V., Potapov A.S. Problems of the Pc1 magnetospheric wave theory. A review. Solar-Terrestrial Physics. 2019. Vol. 5. Iss. 3. P. 87-92. DOI: 10.12737/stp-53201910. 